\title{
Bioreactance and Apelin in the Management of Severe Hyponatremia
}

\author{
Karin Olsson" ${ }^{1,2}$, Magnus Löndahl'1,2, Olle Melander ${ }^{1,3}$, Per Katzman",2 \\ ${ }^{1}$ Department of Clinical Sciences, Lund University, Lund, Sweden \\ ${ }^{2}$ Department of Endocrinology, Skane University Hospital, Lund, Sweden \\ ${ }^{3}$ Department of Emergency and Internal Medicine, Skane University Hospital, Malmö, Sweden \\ Email: ^Karin.C.Olsson@skane.se
}

How to cite this paper: Olsson, K., Löndahl, M., Melander, O. and Katzman, P. (2021) Bioreactance and Apelin in the Management of Severe Hyponatremia. Open Journal of Emergency Medicine, 9, 1-10. https://doi.org/10.4236/ojem.2021.91001

Received: March 2, 2021

Accepted: March 23, 2021

Published: March 26, 2021

Copyright ( 2021 by author(s) and Scientific Research Publishing Inc. This work is licensed under the Creative Commons Attribution International License (CC BY 4.0).

http://creativecommons.org/licenses/by/4.0/ (c) (i) Open Access

\begin{abstract}
Hyponatremia is a severe electrolyte disturbance associated with substantial morbidity and mortality. It often poses a diagnostic and therapeutic challenge. Accurate assessment of patient fluid-volume status is central to effective management. This pilot study aimed to evaluate the usefulness of the Cheetah NICOM bioreactance system and apelin in early differentiation between hypo- and euvolemia in patients with severe hyponatremia. Methods: Patients $>50$ years of age with a serum sodium $\leq 125 \mathrm{mmol} / \mathrm{L}$ were eligible for inclusion after written informed consent. Blood- and urine analyses of cardiovascular load (NT-proBNP), osmotic stress (copeptin, apelin, osmolality, sodium), mineralocorticoid status (aldosterone, renin) and sympathetic activity (methoxycathecholamines) were analysed at baseline and after isotonic sodium chloride infusion. Bedside bioreactance examination was used to visualise parameters, including stroke volume before and after passive leg raise test. Classification of volume status was made retrospectively blinded for biomarker and bioreactance results. Results: 8 patients (4 hypovolemic and 4 euvolemic), 79 years old, median plasma sodium $120 \mathrm{mmol} / \mathrm{L}$ were included. At the Emergency Department volume status was misclassified in all hypoand in 2 of 4 euvolemic patients. Apelin was significantly higher in hypovolemic patients ((299 vs. $175 \mathrm{ng} / \mathrm{ml}), \mathrm{p}=0.021)$. All hypovolemic, but none of the euvolemic, patients had a level above $250 \mathrm{ng} / \mathrm{ml}$. Copeptin did not differ between groups. All patients in the hypovolemic group increased their stroke volume after passive leg raise. Conclusions: Apelin seems to be a promising future biomarker in the early management of severe hyponatremia. Bioreactance measurements may offer a supplement to bedside evaluation of volume status.
\end{abstract}




\section{Keywords}

Hyponatremia, Apelin, Vasopressin

\section{Introduction}

Hyponatremia is a severe electrolyte disturbance with multifactorial aetiology, associated with substantial morbidity and mortality [1] [2]. However, despite being the most common water balance disorder, it often poses a diagnostic and therapeutic challenge, and the clinical management of hyponatremia is still not optimal [3] [4]. After excluding non-hypotonic causes of hyponatremia, diagnosis at the emergency department is usually guided by assessed patient volume status or urinary sodium (U-sodium) concentration. Patient volume status is difficult to determine accurately even for experienced doctors and urinary sodium might be challenging to obtain and is affected by renal function and use of diuretics. At the same time the determination of volume status in hyponatremia is crucial for choosing the correct treatment regime. This study examines bioreactance measurement and plasma apelin levels for accurate, objective differentiation between hypo- and euvolemia in patients with severe hyponatremia.

Vasopressin is a key hormone in the regulation of plasma osmolality and fluid volume status but is difficult to measure. Copeptin, the C-terminal part of the prohormone, can be used as a surrogate for vasopressin levels [5]. Previous studies have indicated that the analysis of copeptin can be useful in the diagnosis of primary polydipsia but cannot reliably differentiate between other causes of hyponatremia [6]. Even though copeptin levels are lowest in primary polydipsia and highest in hypovolemic hyponatremia the large overlap in copeptin levels prevents use in differential diagnosis of hyponatremia. It cannot distinguish between hypo- and euvolemic patients [7].

Apelin, an endogenous peptide, is also involved in the regulation of plasma osmolality and fluid volume status. It regulates vascular tone, cardiac contractile function and fluid balance [8] [9]. Apelin counteracts vasopressin by inhibiting the phasic electric activity of arginine vasopressin neurons, reducing vasopressin blood levels and increasing free water renal excretion. Increased plasma osmolality simultaneously raises plasma vasopressin levels and decreases plasma apelin levels in control subjects. Conversely, decreased plasma osmolality reduced plasma vasopressin levels and rapidly increased plasma apelin levels [10] [11]. Apelin has not previously been evaluated in the differential diagnosis of hyponatremia.

The Cheetah NICOM system is a bedside bioreactance measurement device designed to visualise the hemodynamic status [12]. It has been used in intensive care units for non-invasive monitoring of sepsis patients for early differentiation between hypotension that will respond to aggressive fluid infusion versus the need for inotropic drugs [13].

The aims of this pilot study were to evaluate the usefulness of bioreactance 
measurement and plasma apelin levels in early differentiation between hypoand euvolemia in patients with severe hyponatremia.

\section{Methods}

\subsection{Participants}

This single-centre pilot study was performed at Skane University Hospital, Sweden (June 2015-Dec 2016). Patients were recruited from the Department of Emergency Medicine. The study was approved by the Regional Ethics Committee, Lund, Sweden and was performed according to the Declaration of Helsinki.

Patients admitted to the Emergency Department at Skane University Hospital, Lund, Sweden, older than 50 years of age with a serum sodium concentration of $\leq 125 \mathrm{mmol} / \mathrm{L}$ and able to give a written informed consent were eligible for inclusion. Patients with seizures, uncompensated heart failure, eGFR $<30 \mathrm{ml} / \mathrm{min}$, systolic blood pressure $<90 \mathrm{mmHg}$, ongoing gastrointestinal losses, or a haemoglobin concentration below $100 \mathrm{~g} / \mathrm{L}$ were excluded, as were patients who had received an intravenous infusion of sodium chloride before inclusion.

\subsection{Procedure}

After examination in the Emergency Room, blood- and spot urine analyses were taken according to standard clinical practice [14]. If hyponatremia was detected, eligible patients were informed about the study by the principal investigator and written informed consent was obtained before any study-related procedure was done. Before transferral to an intermediary care ward, blood- and spot urinary samples were taken and stored for later analysis, the physician at the Emergency Department assessed patient volume status, and the principal investigator evaluated volume status with the Cheetah NICOM system. Routine treatment was then initiated with $1000 \mathrm{ml}$ of isotonic sodium chloride over 10 hours, and drugs suspected of causing or aggravating hyponatremia were withheld. Plasma sodium, osmolality levels and biomarkers were analysed at $4 \mathrm{~h}, 12 \mathrm{~h}, 24 \mathrm{~h}$ and daily until discharge.

Classification of volume status was made retrospectively after patient discharge based on available information in patient records, routine blood- and urine analyses as well as response to treatment [14]. Bioreactance measurements, as well as biomarkers measured in stored samples, were not available for the expert.

Plasma and urine samples were immediately frozen and stored at $-80^{\circ} \mathrm{C}$ until analysis, including biomarkers of cardiovascular load (NT-proBNP, osmotic stress (copeptin, apelin), and mineralocorticoid status (aldosterone, renin). The sympathetic activity was elucidated in urine collected in 4 - 12-hour fractions using methoxycatecholamine to creatinine ratio. NT-proBNP was determined using the Dimension RxL N-BNP (Dade-Behring, Marburg, Germany) [15]. The reference range for the NT-proBNP assay is $<300 \mathrm{ng} / \mathrm{L}$ in patients $>60$ years, with an interassay CV of $4.6 \%$ [16]. Copeptin was measured using a fully automated immunoassay system (Kryptor, Thermo Fisher, Henningsdorf, Germany) 
[5]. Median copeptin in 359 healthy individuals in previous investigations was $4.2 \mathrm{pmol} / \mathrm{L}$, with $2.5^{\text {th }}$ and $97.5^{\text {th }}$ percentiles being 1.7 and $11.3 \mathrm{pmol} / \mathrm{L}$, respectively. The interassay CV at $4.2 \mathrm{pmol} / \mathrm{L}$ is $14 \%$ and $9 \%$ at $11.25 \mathrm{pmol} / \mathrm{L}$ [6]. Apelin were analysed using ELISA (Ray Biotech Inc, Norcross GA, USA.) The interassay $\mathrm{CV}$ is $<15 \%$. Median Apelin in 35 healthy individuals with a median age of 55 years was $325 \pm 152 \mathrm{pg} / \mathrm{ml}$ [17] [18]. Blood and urine samples for evaluation of electrolytes, creatinine, osmolality, thyroid hormones, cortisol, and ACTH were analysed using standard procedures at our clinical laboratory (Fiske model 210, Micro-Osmometer. Cobas 6000/8000 ROCHE, Basel, Switzerland). Methoxycatecholamines were analysed using liquid chromatography-tandem mass spectrometry method [19] [20].

Bioreactance measurements were performed using the NICOM system (Cheetah Medical, Portland, OR, USA). The device is connected to the patient with four double-electrodes on the chest wall. An alternating electric current pass between the outer electrodes and the inner electrodes senses the resulting voltage signal. Comparison of the phase shift is proportional to aortic flow. The first examination was undertaken at the Emergency Department, and these baseline values, including heart rate, stroke volume, mean arterial pressure and blood pressure, were registered with patients in a $45^{\circ}$ semi-recumbent position. The device then calculated cardiac output and total peripheral resistance. A passive leg raise test was performed by lowering the head to a flat position and elevating the legs to $45^{\circ}$. An increase in stroke volume index (SVI $=$ SV/body surface area) $\geq 10 \%$ was considered a sign of fluid responsiveness i.e. hypovolemia [12].

Since the hallmark of SIADH is an inappropriate secretion of vasopressin related the sodium level, we calculated a ratio for this inappropriate response by dividing copeptin with plasma sodium.

\subsection{Statistics}

Data were analysed using SPSS statistical software (version 25, SPSS Inc, Chicago Ill, USA).

Data are given as median (range) and percentages. Mann-Whitney U-test was used to compare continuous variables between groups, and Fisher's exact test to compare categorical variables. A p-value $<0.05$ was considered as statistically significant.

\section{Results}

In total, 8 participants, six women, and two men, with a median age of 79 (63 88 ) years, were included in this pilot study. Their baseline sodium level was 120 (107 - 123) $\mathrm{mmol} / \mathrm{L}$, and all but one was taking drugs tentatively associated with hyponatremia, beta-blockers $(n=4)$, diuretics $(n=3)$, antiepileptic drugs $(n=$ $1)$, and selective serotonin uptake inhibitors $(\mathrm{n}=1)$ (Table 1$)$.

Four of the patients were classified bedside at admission as hypovolemic, and four euvolemic. 
Table 1. Patient baseline characteristics.

\begin{tabular}{lc}
\hline & Median, $\%(\mathbf{n}=\mathbf{8})$ \\
\hline Age (yrs) & $75 \%$ \\
Gender (female) & \\
Morbidities: & $38 \%$ \\
$\quad$ Heart failure & $63 \%$ \\
Hypertension & $25 \%$ \\
Cerebrovascular disease & $13 \%$ \\
Epilepsy & $13 \%$ \\
Depression & $25 \%$ \\
Diabetes mellitus & $13 \%$ \\
Previous cancer & \\
Drug treatment: & $38 \%$ \\
Diuretics & $13 \%$ \\
SSRI & $13 \%$ \\
Antiepileptic drugs & $50 \%$ \\
Betablockers & \\
\hline
\end{tabular}

The emergency physicians' determinations, based on all available information at the time of patient transferral to an intermediary care ward, were incorrect in all four cases in the hypovolemic group, and in two of the four cases in the euvolemic group.

Comparisons of biochemical and bioreactance measures between hypovolemic and euvolemic patients are shown in Figure 1. Urine osmolality and urine sodium concentration were higher in the euvolemic group (U-Osm $537 \mathrm{mOsm} / \mathrm{kg}$ (290 - 600) versus $236 \mathrm{mOsm} / \mathrm{kg}(204-269), \mathrm{p}=0.317$ and U-Na $82 \mathrm{mmol} / \mathrm{L}(44$ - 149) vs. $24 \mathrm{mmol} / \mathrm{L}(20-34), \mathrm{p}=0.180$, respectively) (Table 2).

Stroke volume, stroke volume index, cardiac output, mean atrial pressure and thoracic fluid content were similar and not statistically different between the two groups (data not shown). All patients in the hypovolemic group increased their stroke volume after passive leg raise. Compared to the euvolemic group, total peripheral resistance index (TPRI) decreased more after passive leg raise in the hypovolemic than the euvolemic group $(-20(-28-(-7))$ vs. $-1(15-46), \mathrm{p}=0.043)$.

At baseline, apelin was significantly higher in the hypovolemic group (299 (265 - 433) vs. 175 (147 - 228), $\mathrm{p}=0.021)$, and apelin concentration was $>250$ $\mathrm{ng} / \mathrm{ml}$ in all hypovolemic patients. In all euvolemic patients, the apelin concentration was below this level (Figure 1).

Copeptin was similar between groups, but the apelin/copeptin ratio significantly differed (48.8 (22.1 - 217.1) vs. $18.5(14.9-24.4), \mathrm{p}=0.043)$.

Copeptin to plasma sodium ratios at admission did not statistically significantly differ between the hypovolemic and euvolemic groups. 

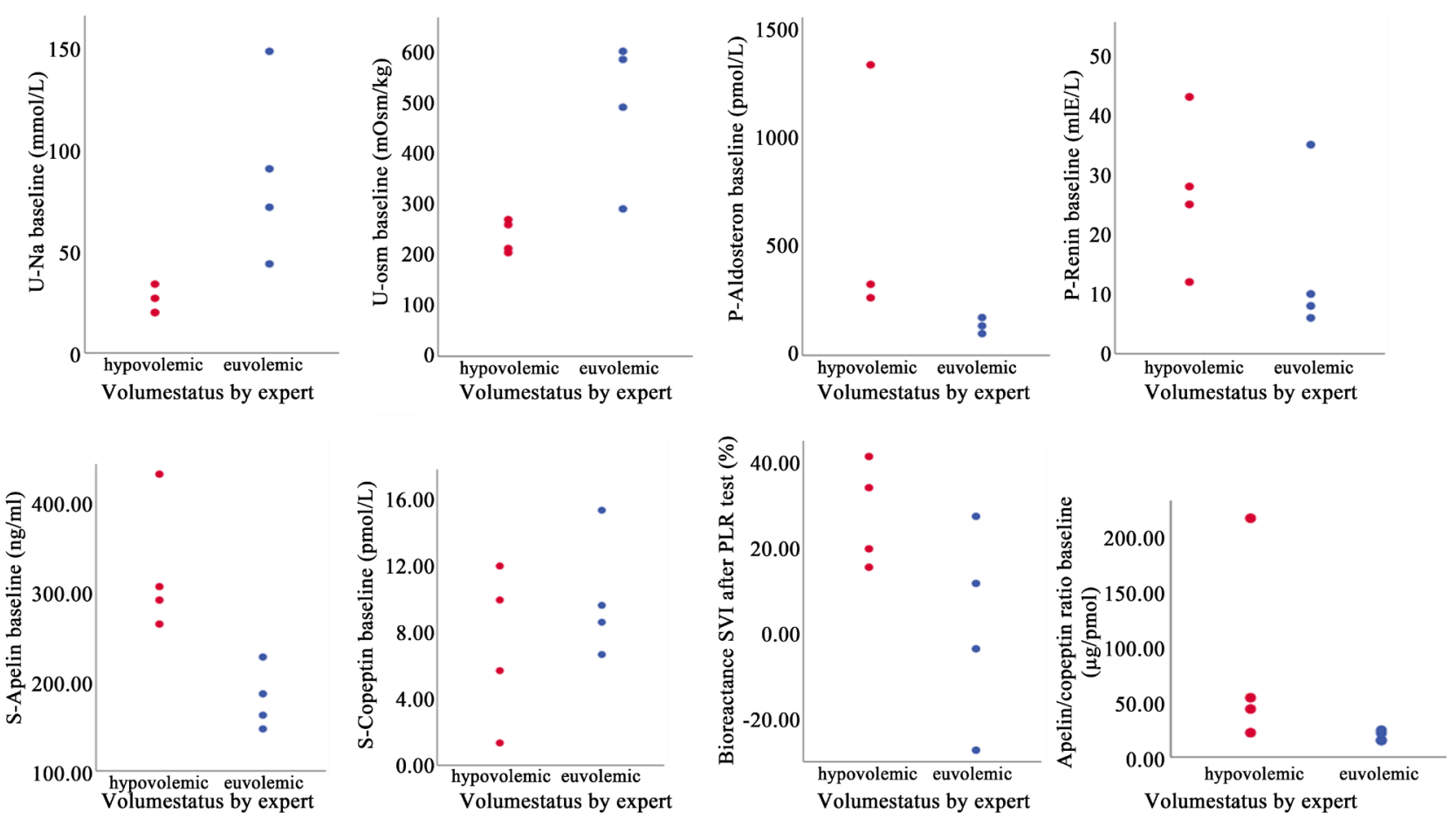

Figure 1. Baseline biomarker levels in patients with hypo- versus euvolemic hyponatremia. Bioreactance analysis of stroke volume index after passive leg raise test.

Table 2. Hypovolemia vs Euvolemia.

\begin{tabular}{|c|c|c|c|}
\hline & $\begin{array}{c}\text { Hypovolemia }(\mathrm{n}=4) \\
\text { Value (range min-max) }\end{array}$ & $\begin{array}{c}\text { Euvolemia }(\mathrm{n}=4) \\
\text { Value (range min-max) }\end{array}$ & $\mathrm{p}$-value \\
\hline Age (years) & $76(63-88)$ & $80(70-86)$ & 0.564 \\
\hline Gender (female) & 4 & 2 & 0.105 \\
\hline BP systolic (mmHg) & $145(130-169)$ & $158(150-200)$ & 0.191 \\
\hline BP diastolic (mmHg) & $76(70-80)$ & $103(74-110)$ & 0.081 \\
\hline Heart rate (beats/min) & $79(60-92)$ & $79(70-87)$ & 1.000 \\
\hline $\mathrm{TSH}(\mathrm{mU} / \mathrm{L})$ & $1.3(1-4)$ & $1.4(1.0-37.0)$ & 0.663 \\
\hline $\mathrm{T} 4(\mathrm{mU} / \mathrm{L})$ & $15(12-18)$ & $20(2-27)$ & 0.309 \\
\hline $\mathrm{T} 3(\mathrm{mU} / \mathrm{L})$ & $3.7(3-5)$ & $3.4(1-4)$ & 0.564 \\
\hline Cortisol $(\mathrm{mmol} / \mathrm{L})$ & $421(191-821)$ & $540(283-582)$ & 0.564 \\
\hline ACTH (pmol/L) & $3.6(2-12)$ & $5.1(3-15)$ & 0.289 \\
\hline $\mathrm{Hb}(\mathrm{g} / \mathrm{L})$ & $122(102-164)$ & $140(128-142)$ & 0.386 \\
\hline Potassium (mmol/L) & $3.9(2.9-4.5)$ & $4.2(4.0-4.5)$ & 0.189 \\
\hline Creatinine $(\mu \mathrm{mol} / \mathrm{L})$ & $101(52-153)$ & $69(45-92)$ & 0.248 \\
\hline Glucose (mmol/L) & $6.0(5-13)$ & $6.5(5.8-8.0)$ & 0.564 \\
\hline CRP (mg/L) & $3(1-6)$ & $2(1-5)$ & 0.663 \\
\hline P-osm (mOsm/kg) & $263(261-288$ & $251(237-277)$ & 0.146 \\
\hline U-osm (mOsm/kg) & $236(204-269)$ & $537(290-600)$ & 0.021 \\
\hline $\mathrm{U}-\mathrm{Na}(\mathrm{mmol} / \mathrm{L})$ & $24(20-34)$ & $82(44-149)$ & 0.020 \\
\hline $\mathrm{U}-\mathrm{K}(\mathrm{mmol} / \mathrm{L})$ & $24(23-36)$ & $31(19-46)$ & 0.724 \\
\hline U-Methoxynorepinephrine/creatinine & $291(191-430)$ & $199(168-674)$ & 0.386 \\
\hline U-Methoxyepinephrine/creatinine & $104(54-219)$ & $141(111-211)$ & 0.564 \\
\hline
\end{tabular}


Cortisol, ACTH, TSH, T4, T3, glucose, CRP, metoxycatecholamines and baseexcess did not differ between groups, and all values were within the reference ranges. Pro-BNP did not differ between groups (451 (140 - 889) vs. 528 (174 1109), n.s.).

A trend towards shorter hospital stay in the hypovolemic group was seen (3 (2 - 10) vs. 7 (3 - 17) days, n.s.).

\section{Discussion}

Optimal management of hyponatremia is fundamentally based on the patient's volume status [14]. However, at the emergency, clinical examination, as well as routine blood and urine samples, seem to be of unpredictable value, which has been confirmed in our as well as previous studies [1] [3] [4]. Accordingly, the present golden standard of volume status at admission is usually done in retrospect by experienced clinicians, based on analysis of clinical and laboratory findings before and following treatment [14] [21].

In the present study, we aimed to improve early bedside evaluation of volume status using biomarkers and a simple test of bioreactance, which mirrors stroke volume before and after "self transfusion" of fluid following passive leg raise [12]. Our findings following this test were in concert with expert opinion in six of the eight patients. To our knowledge, this method has not previously been studied in severe hyponatremic patients, and our outcome suggests further studies to validate our findings. Bioreactance may aid in determination of volume status in hyponatremic patients and perhaps also be an instrument to further understanding of the complex relationship between volume status and sodium in multifactorial hyponatremia.

Apelin levels were significantly higher in hypo- than euvolemic, hyponatremic patients, and none of our studied hypovolemic patients displayed a value below $250 \mathrm{nmol} / \mathrm{l}$. This may mirror that a normal physiological response to hyponatremia or hypovolemia requires crosstalk between vasopressin and apelin, not present in SIADH patients [11].

Vasopressin levels, evaluated by analysing copeptin were similar in hypo- and euvolemic hyponatremia, confirming findings in previous studies [6] [7].

Regarding hypovolemia, our findings are in concert with those of a previous study, where Urwyler et al. found higher plasma apelin levels in patients with nephrogenic diabetes insipidus, however, no previous study has found differences in apelin between hypo- and euvolemic hyponatremia [22]. The outcome of our small pilot study, thus indicates that apelin could be a useful marker for differentiating between hypo- and euvolemia in the early management of hyponatremia.

In a routine clinical management perspective, differentiation based on apelin concentration may be preferable to bioreactance measurements, as the former is easily accessible by a single blood sample. In contrast, a bioreactance measurement not only is more time-consuming but also requires a device and individual 
skills to handle it.

The main limitation of our study is the number of patients included. However, a positive outcome of the hypothesis in a small prospective study might indicate a high clinical relevance, which should be confirmed in further studies.

In conclusion, our small pilot study indicates that apelin seems to be a promising future tool in the early management of severe hyponatremia. Bioreactance measurements may offer a supplement to bedside evaluation of volume status.

\section{Acknowledgements}

We would like to thank R.N. Margit Bergström, Widet Gallo and the staff and patients in the Emergency Department, Skane University Hospital, for assistance and cooperation.

\section{Funding}

Funding was obtained from Lund University Infrastructure Grant 2020-2024, the Swedish Research Council; Region Skane-ALF/ Skane University Hospital, Knut and Alice Wallenberg Foundation; the Göran Gustafsson Foundation; the Swedish Heart- and Lung Foundation, European Research Council ERC-AdG-2019-885003 and the Novo Nordisk Foundation. Investigator initiated grant from Otsuka Pharmaceuticals.

\section{Disclosure Summary}

Dr. Olsson has served as a consultant in educational activities arranged by Otsuka Pharma Scandinavia AB. The study was partly financed by Investigator Initiated Study Agreement with Otsuka pharmaceuticals.

\section{Conflicts of Interest}

The authors declare no conflicts of interest regarding the publication of this paper.

\section{References}

[1] Olsson, K., Öhlin, B. and Melander, O. (2013) Epidemiology and Characteristics for Hyponatremia in the Emergency Department. European Journal of Internal Medicine, 24, 110-116. https://doi.org/10.1016/j.ejim.2012.10.014

[2] Peri, A. (2019) Morbidity and Mortality of Hyponatremia. Disorders of Fluid and Electrolyte Metabolism, 52, 36-48. https://doi.org/10.1159/000493235

[3] Chung, H.M., Kluge, R., Schrier, R.W. and Anderson, R.J. (1987) Clinical Assessment of Extracellular Fluid Volume in Hyponatremia. American Journal of Medicine, 83, 905-908. https://doi.org/10.1016/0002-9343(87)90649-8

[4] Musch, W., Thimpont, J., Vandervelde, D., Verhaeverbeke, I., Berghmans, T. and Decaux, G. (1995) Combined Fractional Excretion of Sodium and Urea Better Predicts Response to Saline in Hyponatremia than Do Clinical and Biochemical Parameters. American Journal of Medicine, 99, 348-355. https://doi.org/10.1016/S0002-9343(99)80180-6 
[5] Morgenthaler, N.G., Struck, J., Alonso, C. and Bergmann, A. (2006) Assay for the Measurement of Copeptin, a Stable Peptide Derived from the Precursor of Vasopressin. Clinical Chemistry, 52, 112-119. https://doi.org/10.1373/clinchem.2005.060038

[6] Fenske, W., Stork, S., Blechschmidt, A., Maier, S.G., Morgenthaler, N.G. and Allolio, B. (2009) Copeptin in the Differential Diagnosis of Hyponatremia. Journal of Clinical Endocrinology and Metabolism, 94, 123-129.

https://doi.org/10.1210/jc.2008-1426

[7] Olsson, K., Enhörning, S., Öhlin, B. and Melander, O. (2016) Hyponatremia in the Emergency Department: Could Biomarkers Help in Diagnosis and Treatment? Open Journal of Emergency Medicine, 4, 11-22.

https://doi.org/10.4236/ojem.2016.41003

[8] Tatemoto, K., Hosoya, M. and Habata, Y. (1998) Isolation and Characterization of a Novel Endogenous Peptide Ligand for the Human APJ Receptor. Biochemical and Biophysical Research Communications, 251, 471-476. https://doi.org/10.1006/bbrc.1998.9489

[9] Antuschevich, H. and Mojcik, M. (2018) Review: Apelin in Disease. Clinica Chimica Acta, 483, 241-248. https://doi.org/10.1016/j.cca.2018.05.012

[10] Azizi, M., Iturrioz, X., Blanchard, A., Peyrard, S., De Mota, N. and Chartrel, N. (2008) Reciprocal Regulation of Plasma Apelin and Vasopressin by Osmotic Stimuli. Journal of the American Society of Nephrology, 19, 1015-1024. https://doi.org/10.1681/ASN.2007070816

[11] Flahault, A., Couvineau, P., Alvear-Perez, R., Iturrioz, X. and Llorens-Cortes, C. (2017) Role of the Vasopressin/Apelin Balance and Potential Use of Metabolically Stable Apelin Analogs in Water Metabolism Disorders. Frontiers in Endocrinology, 8, 120. https://doi.org/10.3389/fendo.2017.00120

[12] Marik, P.E., Levitov, A., Young, A. and Andrews, L. (2013) The Use of Bioreactance and Carotid Doppler to Determine Volume Responsiveness and Blood Flow Redistribution Following Passive Leg Raising in Hemodynamically Unstable Patients. Chest, 143, 364-370. https://doi.org/10.1378/chest.12-1274

[13] Latham, H.E., Bengtson, C.D., Satterwhite, L., Stites, M., Subramaniam, D.P., Chen, J.C. and Simpson, S.Q. (2017) Stroke Volume Guided Resuscitation in Severe Sepsis and Septic Shock Improves Outcomes. Journal of Critical Care, 42, 42-46. https://doi.org/10.1016/j.jcrc.2017.06.028

[14] Hoorn, E.J. and Zietse, R. (2017) Diagnosis and Treatment of Hyponatremia: Compilation of the Guidelines. Journal of the American Society of Nephrology, 5, 1340-1349. https://doi.org/10.1681/ASN.2016101139

[15] Di Serio, F., Ruggieri, V., Varraso, L., De Sario, R., Mastrorilli, A. and Pansini, N. (2005) Analytical Evaluation of the Dade Behring Dimension RxL Automated N-Terminal proBNP (NT-proBNP) Method and Comparison with the Roche Elecsys 2010. Clinical Chemistry and Laboratory Medicine, 43, 1263-1273. https://doi.org/10.1515/CCLM.2005.217

[16] Januzzi, J., Kimmenade, R., Lainchbury, J., Bayes-Genis, A., Ordonez-Lianos, J. and Santalo-Bel, M. (2006) NT-proBNP Testing for Diagnosis and Short-Term Prognosis in Acute Destabilized Heart Failure: An International Pooled Analysis of $1256 \mathrm{~Pa}$ tients. European Heart Journal, 27, 330-337. https://doi.org/10.1093/eurheartj/ehi631

[17] Liakos, C., Sanidas, E., Perrea, D., Grassos, C., Chantziara, V., Viniou, N., Barabetseas, J. and Papadopoulos, D. (2016) Apelin and Visfatin Plasma Levels in Healthy Individuals with High Normal Blood Pressure. American Journal of Hypertension, 
29, 549-552. https://doi.org/10.1093/ajh/hpv136

[18] Karakoç, A., Sahin, A., Polat, E.S., Aliyev, E., Yildirim, A., Bakan, N. and Dokumacioglu, E. (2016) Serum Apelin and ADMA Levels in Type 2 Diabetics with and without Vascular Complications. Diabetes \& Metabolic Syndrome: Clinical Research \& Reviews, 10, S106-S109. https://doi.org/10.1016/j.dsx.2016.03.005

[19] Taylor, R. and Singh, R. (2002) Validation of Liquid Chromatography-Tandem Mass Spectrometry Method for Analysis of Urinary Conjugated Metanephrine and Normetanephrine for Screening of Pheochromocytoma. Clinical Chemistry, 48, 533-539. https://doi.org/10.1093/clinchem/48.3.533

[20] Quante, S., Boretti, F.S., Kook, P.H., Mueller, C., Schellenberg, S., Zini, E., Sieber-Ruckstuhl, N. and Reusch, C.E. (2010) Urinary Catecholamine and Metanephrine to Creatinine Ratios in Dogs with Hyperadrenocorticism or Pheochromocytoma, and in Healthy Dogs. Journal of Veterinary Internal Medicine, 24, 1093-1097. https://doi.org/10.1111/j.1939-1676.2010.0578.x

[21] Hoorn, E.J., Lindemans, J. and Zietse, R. (2006) Development of Severe Hyponatraemia in Hospitalized Patients: Treatment-Related Risk Factors and Inadequate Management. Nephrology Dialysis Transplantation, 21, 70-76.

https://doi.org/10.1093/ndt/gfi082

[22] Urwyler, S.A., Timper, K., Fenske, W., de Mota, N., Blanchard, A. and Kühn, F. (2016) Plasma Apelin Concentrations in Patients with Polyuria-Polydipsia Syndrome. The Journal of Clinical Endocrinology \& Metabolism, 101, 1917-1923. https://doi.org/10.1210/jc.2016-1158 